\title{
Resistencia de concreto con agregado de bloque de arcilla triturado como reemplazo de agregado grueso
}

\author{
Resistance of concrete with aggregate of clay block crushed \\ as replacement of aggregate thick
}

\author{
Luis Ángel Moreno Anselmi ${ }^{1 *} \quad$ Miguel Ángel Ospina García ${ }^{1} \quad$ Kelly Andrea Rodríguez Polo ${ }^{1}$ \\ Recibido 29 de noviembre de 2017, aceptado 13 de septiembre de 2018 \\ Received: November 29, 2017 Accepted: September 13, 2018
}

\begin{abstract}
RESUMEN
El presente artículo hace una revisión del estado del arte de las investigaciones sobre la resistencia de concretos con el uso de bloque de arcilla triturado como sustitución total del agregado grueso, se ha analizado los efectos del uso de este material desde las siguientes características: la durabilidad, resistencia a la compresión, la porosidad, el módulo de elasticidad, la tensión indirecta del concreto. También se presenta un caso de estudio de un establecimiento penitenciario donde se hace el análisis de resistencia de la compresión sobre una muestra de una columna interior del establecimiento. La revisión muestra que son insuficientes los resultados anunciados sobre el efecto neto del uso de triturado de bloque de arcilla como agregado grueso en las diferentes propiedades del concreto tanto en su estado fresco como endurecido. También se establece que los concretos derivados con agregados reciclados presentan menor resistencia a la compresión que las mezclas tradicionales.
\end{abstract}

Palabras clave: Bloque de arcilla triturado, agregado grueso, concreto reciclado.

\begin{abstract}
This article makes a review of the state of the art of research on concrete resistance with the use of clay block crushed as a total replacement of the coarse aggregate, has analyzed the effects of the use of this material from the Following characteristics: Durability, compression resistance, porosity, modulus of elasticity, indirect stress of concrete. Also, here is a case study of a penitentiary establishment where the compression resistance analysis is performed on a sample of an interior column of the establishment. The review shows that the results announced on the net effect of the use of clay block grinding as a coarse aggregate in the different properties of the concrete both in their fresh and hardened state are insufficient. It is also established that concrete derivatives with recycled aggregates have less resistance to compression than traditional mixes.
\end{abstract}

Keywords: crushed clay block, coarse aggregate, recycled concrete.

\section{INTRODUCCIÓN}

Existe una necesidad de implementar mejoras que permitan reducir el impacto ambiental de la producción de concretos y sus componentes [1-3].
Extensivas investigaciones en los últimos 15 años han sido realizadas con la finalidad de evaluar el uso de residuos de la demolición como reemplazo de los agregados naturales [4], con la finalidad de reducir el impacto ambiental que generan estos y

\footnotetext{
1 Universidad Militar Nueva Granada, Km 2 Vía Cajicá-Zipaquirá, Edificio Facultad de Estudios a Distancia Cajicá. E-mail: luis.moreno@unimilitar.edu.co; miguel.ospina@unimilitar.edu.co; tmp.kelly.rodriguez@unimilitar.edu.co

* Autor de correspondencia. E-mail: luis.moreno@unimilitar.edu.co
} 
los costos productivos [5] particularmente, el uso de agregados, producto del reciclado de demoliciones, disminuye la acumulación de residuos sólidos en escombreras evita la explotación innecesaria de las canteras [6].

La implementación de residuos de construcción, en la producción de concreto tiene como limitación la variabilidad en la composición de los mismos [7]. La literatura revisada sugiere que la durabilidad y las propiedades del concreto elaborado con agregados reciclado de la construcción RAC (por sus siglas en inglés: (Recycled Aggregate Concrete) son generalmente menores que las mezclas concreto tradicional [4].

Adicionalmente, aunque al emplear residuos de la construcción, la separación del ladrillo triturado de arcilla del concreto y/o mortero presenta una dificultad operacional e implica unos costos importantes [5]. Sin embargo, es necesario estimar el efecto del uso de bloques de arcilla cocida como agregado del concreto con el fin de fundamentar diferentes ensayos específicos para este material reciclado.

En este artículo se presenta una revisión documental sobre la resistencia de concretos con el uso de bloque de arcilla triturado como reemplazo total del agregado grueso únicamente con la finalidad de proveer una síntesis de los resultados, visualizar la posibilidad de establecer el uso de la misma en zonas donde la disponibilidad de agregados naturales sea limitada y/o poco práctica $\mathrm{y}$, discutir sobre la posibilidad de alcanzar resistencias de diseño específicas que puedan requerir los diferentes proyectos de ingeniería. Adicionalmente, se presentan resultados del comportamiento mecánico de este tipo de mezclas obtenidas de un establecimiento penitenciario el cual tiene alrededor de 40 años demostrando su aplicabilidad.

\section{METODOLOGÍA}

Se realiza un análisis comparativo de los diferentes estudios sobre el uso de triturado de bloque de arcilla haciendo énfasis en la resistencia a la compresión. Para su desarrollo la metodología corresponde a una investigación documental, con el fin de evaluar las propiedades al usar este material en el campo de la construcción. Esta posibilidad se basa en la premisa de poder desarrollar propiedades mecánicas específicas con este tipo de concreto. Por otra parte, aunque existen algunas revisiones recientes en materia de los usos de residuos de la construcción $[4,7]$, estas no destacan el efecto del uso del bloque de arcilla como remplazo del agregado grueso.

\section{Uso de agregado reciclado de bloque de arcilla: Una revisión}

El uso de bloque de arcilla reciclado es recomendable para muchas aplicaciones donde la resistencia térmica, el costo y los aspectos ambientales son fundamentales [1]. Sin embargo, una serie de investigaciones concluyen en limitar el uso de este agregado, por ejemplo, Adamson et al. [8] recomienda no reemplazar el agregado natural con ladrillo de arcilla triturado en concretos reforzados con acero. En este último estudio se encontró que la resistencia a la penetración de los cloruros se reduce en la medida que se incrementa el contenido del triturado, por lo tanto sería positivo usar áridos de ladrillo de arcilla, al reducir la penetración de estos materiales corrosivos.

Hay investigaciones que sugieren que el uso del ladrillo de arcilla triturado con otros materiales tiene la posibilidad de incrementar las propiedades mecánicas de éste. Por ejemplo, [9] encuentran que al reemplazar el $100 \%$ de los agregados gruesos por residuos cerámicos (mezcla de ladrillos cerámicos; azulejo blanco y porcelana sanitaria), la resistencia a la compresión se incrementa en un $11 \%$.

Debieb and Kenai [10] examinan la posibilidad de usar el triturado de bloque de arcilla como agregado fino y grueso usando diferentes porcentajes de reemplazos $(25,50,75$ y 100\%) a los agregados tradicionales. Los resultados mostraron que es posible desarrollar concretos con triturado de ladrillo de arcilla cocida con características similares a los concretos tradicionales mientras se limite a un $25 \%$ y $50 \%$ la cantidad de agregado grueso y fino, respectivamente. Aliabdo et al. (2014) [1] realizó ensayos de compresión en 26 mezclas de concreto incorporando diferentes proporciones de escombros de ladrillo triturado como agregado para presentar un estudio comparativo.

Estos resultados confirmaron que el incremento del contenido de agregado reciclado disminuye la resistencia a la compresión de las mezclas. Un 
estudio en las propiedades físicas y mecánicas de concretos elaborados con RAC y triturado de bloque de arcilla cocido CCB (por sus siglas en inglés: (Crushed Clay Bricks) con la finalidad de establecer limitaciones en este tipo de mezclas fue desarrollado por Yang et al. [11]. Este estudió mostro que cuando se reemplaza el $50 \%$ con CCB se afecta la resistencia del concreto progresivamente, a los 7 días se reporta una reducción del $14 \%$ y del $20 \%$ a los 28 días.

Por otra parte, la deformación progresiva del concreto usando triturado de piedra y CCB como agregado grueso fue evaluado por Ahmad and Roy [12]. Dos grados diferentes de concretos fueron evaluados (17.2 y 27.5 MPa) usando un diseño respectivo de mezcla con los dos componentes y fueron evaluados durante un periodo de 50 días. Los resultados mostraron que las mezclas realizadas con triturado de piedra presentan la mayor deformación progresiva. Además, como se incrementa la resistencia del concreto, la diferencia en la deformación progresiva entre los concretos realizados con triturado de piedra y CCB se incrementa.

En Colombia, [13] evalúa el uso de CCB como reemplazo parcial del agregado grueso $(10,20 \mathrm{y}$ $30 \%$ de reemplazo del agregado triturado). Los resultados indican una clara disminución en la resistencia a la compresión cuando se realiza el uso del CCB. Sin embargo, todos los concretos presentaron una resistencia superior a los $21 \mathrm{MPa}$ y la reducción de la capacidad respecto a concreto tradicional (sin reemplazo) varía entre un 2 y $6 \%$. Silva et al. [14] explora la posibilidad de desarrollar concretos autocompactantes usando residuos de demolición de mampostería. Los resultados permiten evidenciar que el uso de este tipo de agregados finos permite obtener unas propiedades mecánicas y de flujo (segregación, exudación y capacidad de relleno) adecuadas para su uso mientras se utilice un porcentaje de reemplazo menor al $30 \%$.

Se realiza un análisis comparativo de los diferentes estudios sobre el uso de triturado de bloque de arcilla haciendo énfasis en la resistencia a la compresión. Para su desarrollo la metodología corresponde a una investigación documental, con el fin de evaluar las propiedades al usar este material en el campo de la construcción. Esta posibilidad se basa en la premisa de poder desarrollar propiedades mecánicas específicas con este tipo de concreto. Por otra parte, aunque existen algunas revisiones recientes en materia de los usos de residuos de la construcción $[4,7]$, estas no destacan el efecto del uso del bloque de arcilla como remplazo del agregado grueso.

\section{Efecto del uso de bloque de arcilla triturado en la durabilidad}

Estudios previos han demostrado que la resistencia a la penetración de los cloruros se reduce en la medida que se incrementa el contenido de bloque de arcilla triturado como agregado, esto se debe a que está directamente relacionado con la alta porosidad y absorción de la arcilla cocida (e.g. Adamson et al. 2015) [8].

\section{Efectos del reemplazo de agregado grueso en la resistencia a la compresión}

En general, las investigaciones muestran que los concretos producidos con agregados reciclados presentan menor resistencia a la compresión que las mezclas tradicionales [7]. Esta reducción es inversamente proporcional a la cantidad de sustitución de [11]. Un incremento lineal en la resistencia a la compresión entre un $20 \%$ y $30 \%$ en concretos con un nivel de sustitución de 50\% y $100 \%$ de agregado grueso por agregado reciclado es reportada por Martínez-Lage et al. [15].

Sin embargo, en un estudio realizado Kallak [16], presenta reducciones en la Resistencia a la compresión hasta de un $87 \%$ para un reemplazo total del agregado grueso por CCB. Yang et al. [11] registro reducciones de un $20 \%$ a los 28 días cuando se reemplaza un $50 \%$ del agregado grueso por CCB. En la Tabla 1 se resumen los resultados de resistencia a la compresión reportados en la literatura y los parámetros más influyentes de la mezclas.

La Tabla 1 muestra que los diferentes estudios han sido realizados con cemento portland ordinario tipo o de uso general, por tanto, investigaciones de las propiedades del concreto con triturado de arcilla con otros tipos de cemento pueden ser explorados.

La relación agua-cemento $\mathrm{A} / \mathrm{MC}$ es considerado uno de los factores más influyente en la resistencia a la compresión del concreto reciclado [17], por tanto, se grafica el porcentaje de reducción de la resistencia a la compresión versus la relación A/MC para los datos presentados en la Tabla 1. 
Tabla 1. Caracterización de los agregados.

\begin{tabular}{|c|c|c|c|c|c|c|}
\hline Investigador & \begin{tabular}{|} 
Contenido de \\
cemento \\
{$\left[\mathrm{kg} / \mathrm{m}^{3}\right]$}
\end{tabular} & $\begin{array}{l}\text { Relación A/C } \\
\text { Patrón/RAC }\end{array}$ & Tipo de cemento & $\begin{array}{c}\text { \% de reemplazo } \\
\text { de agregado } \\
\text { grueso }\end{array}$ & $\begin{array}{c}f^{\prime} c^{*} \\
{[\mathrm{MPa}]}\end{array}$ & $\begin{array}{l}f^{\prime} c^{* *} \\
{[\mathrm{MPa}]}\end{array}$ \\
\hline \multirow{2}{*}{ Aliabdo et al., 2014} & 250 & $0,70 / 0,70$ & \multirow{2}{*}{$\begin{array}{l}\text { Cemento Portland CEM-I } \\
\text { 42,5N (ASTM Tipo I) }\end{array}$} & \multirow{2}{*}{$25,50,75$ у 100} & 22,0 & 16,0 \\
\hline & 350 & $0,5 / 0,50$ & & & 34,0 & 22,5 \\
\hline Adamson et al. 2015 & No reportado & $0,42 / \mathrm{NA}$ & Uso General o Tipo I & 25 y 50 & 46,0 & N/A \\
\hline Debieb and Kenai 2008 & 350 & $0,60 / 0,57$ & $\begin{array}{l}\text { Uso General o Tipo CEM } \\
\text { I-32,5 }\end{array}$ & $25,50,75$ y 100 & 30,0 & 21,5 \\
\hline Kallak 2009 & 250 & $0,47 / 0,75$ & $\begin{array}{l}\text { Cemento Portland }\left(f^{\prime} c \text { a los }\right. \\
3 \text { días de } 23,8 \mathrm{MPa}\end{array}$ & $25,50,75$ y 100 & 30,0 & 3,2 \\
\hline \multirow{3}{*}{ Cabral et al. 2010} & \multirow{3}{*}{$430 * * *$} & $0,46 / 0,46$ & \multirow{3}{*}{$\begin{array}{l}\text { Cemento Portland ( } f^{\prime} c \text { a } \\
\text { los } 3 \text { días de } 39,5 \mathrm{MPa}\end{array}$} & \multirow{3}{*}{50,100} & 46,0 & 30,5 \\
\hline & & $0,60 / 0,60$ & & & 35,0 & 23,5 \\
\hline & & $0,74 / 0,74$ & & & 27,0 & 17,5 \\
\hline Yang et al. 2011 & 435 & $0,47 / 0,47$ & $\begin{array}{l}\text { Cemento Portland Ordi- } \\
\text { nario CEM I BS EN 197-1 }\end{array}$ & 50 & 54,7 & N/A \\
\hline \multirow{2}{*}{ Ahmad \& Roy 2011} & $314 * * *$ & $0,58 / 0,55$ & \multirow{2}{*}{$\begin{array}{l}\text { Cemento Portland Ordi- } \\
\text { nario (Tipo I) }\end{array}$} & 100 & 17,7 & 18,6 \\
\hline & $431 * * *$ & $0,38 / 0,37$ & & 100 & 30,2 & 28,6 \\
\hline Perez-Rojas 2012 & $250 * * *$ & $0,5 / 0,5$ & $\begin{array}{l}\text { Cemento Portland Tipo } \\
\text { I (Argos) }\end{array}$ & 10,20 y 30 & 24,9 & N/A \\
\hline
\end{tabular}

* de concreto patrón (28 días), (N/A = No aplica).

** de concreto con $100 \%$ de reemplazo del agregado grueso (28 días).

*** Approximate value based on design mix described by authors.

En la Figura 1 se puede observar que en una reducción promedio del $30 \%$ puede ser esperada cuando se reemplaza el $100 \%$ de los agregados gruesos por el ladrillo de arcilla triturado. Además, teniendo en cuen- ta que los puntos graficados son para diferentes cantidades de la relación agua cemento, la reducción en la resistencia a la compresión parecen no ser influenciados por la cantidad de cemento usado en la mezcla.

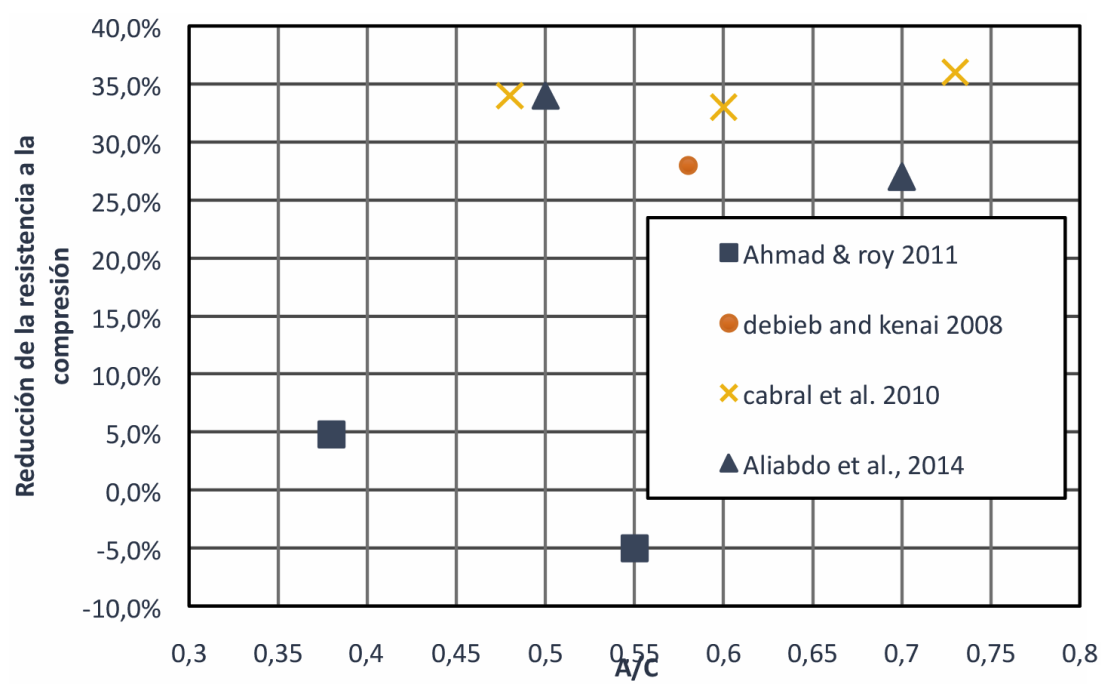

Figura 1. Variación en la resistencia en la compresión cuando se usa ladrillo de arcilla triturado como agregado grueso. 
Los valores reportados por Kallak [16] para el concreto con agregado de arcilla no son graficados ya que no se usó una relación agua/cemento similar que en el concreto patrón $(0,75$ vs 0,47$)$ y por tanto, no pueden ser comparados. Los resultados reportados por Ahmad \& Roy [12] presentan un comportamiento totalmente diferente, es decir, para una relación agua cemento de 0,55 el concreto con trozos de ladrillo presenta una mejor resistencia que el concreto realzado con triturado de piedra.

\section{Efectos del reemplazo de agregado grueso en la porosidad}

La absorción de agua del agregado CCB es mayor que la presentada en los agregados naturales del concreto [8]. Además, se ha reportado que el agregado CCB presenta una absorción de agua mayor al concreto elaborado con la mezcla de variedad de residuos de la construcción [11]. [1] Aliabdo por ejemplo, reporta un incremento en la porosidad en concretos con CCB entre el $14,5 \%$ y $25,0 \%$ para una mezcla con un contenido de cemento de $350 \mathrm{~kg}$ por metro cúbico de concreto y $\mathrm{A} / \mathrm{MC}=0,5 \mathrm{y}$, de 16 a $26 \%$ para un contenido de cemento de $250 \mathrm{~kg}$ por metro cúbico de concreto y $\mathrm{A} / \mathrm{MC}=0,7$.

Sin embargo, otros estudios que reportan cifras aún mayores [10], determinaron que la permeabilidad al agua de las mezclas con CCB se duplica al reemplazar el agregado grueso por ladrillo triturado también han establecido que la permeabilidad de los concretos reciclados puede ser hasta cinco veces mayor que el del concreto con agregados naturales $[10,18]$.

\section{Efectos del reemplazo de agregado grueso en el módulo de elasticidad}

El módulo de elasticidad del concreto reciclado es típicamente menor que el de concretos tradicionales y el uso de agregado CCB como agregado grueso presenta la mayor influencia [7]. Un alto reemplazo de agregado grueso por CCB reduce el módulo de elasticidad significativamente; [1] determinó una reducción en el módulo de elasticidad en concretos con CCB entre 20,0 y 34,5 a $20 \mathrm{MPa}$ para una mezcla con un contenido de cemento de $350 \mathrm{~kg}$ y $\mathrm{A} / \mathrm{C}=0,5 \mathrm{y}$, de 16 a $28 \mathrm{MPa}$ para un contenido de cemento de $250 \mathrm{~kg}$ y A/C $=0,7$. Estas reducciones son típicamente atribuidas a la alta porosidad del bloque de arcilla comparado con los agregados pétreos naturales [8].

\section{Efectos del reemplazo de agregado grueso en la tensión indirecta del concreto}

Reemplazar el agregado grueso tradicional por altas cantidades de CCB, reduce la resistencia a la tención indirecta de manera considerable $[1,16]$. Registró que la relación entre la resistencia a la tención indirecta entre los concretos elaborados con CCB y el concreto tradicional se encuentra entre 0,2 y 1,4 .

\section{Recomendaciones en el reemplazo de agregados gruesos}

Un análisis de la calidad del bloque de arcilla cocida y de su origen debe ser el primer paso cuando se desea incorporar este tipo de residuo de la construcción como sustitución de los agregados naturales de las mezclas de concreto [13]. En estas mezclas se debe tener especial cuidado en los diseños para evitar el agrietamiento y reducir los problemas de durabilidad en refuerzo del concreto [10]. Específicamente, se debe tener en cuenta el cambio en la cantidad de agua demandada y ajustar la relación $\mathrm{A} / \mathrm{C}$ para considerar la alta porosidad y absorción de los agregados reciclados [8].

Diferentes investigadores sugieren limitar la cantidad de agregado reciclado para evitar una pérdida considerable en la resistencia de los concretos reciclados; [1] recomienda usar agregados reciclados CCB como un reemplazo del $25 \%$ y $50 \%$ del agregado grueso natural para mezclas de concreto con $350 \mathrm{y}$ $250 \mathrm{Kg}$ de cemento, respectivamente.

Un límite del 25\% para el agregado grueso y del $50 \%$ para el agregado fino por CCB es propuesto por [10], con la finalidad de obtener propiedades similares a las de un concreto tradicional. En esta misma dirección, [13] propone limitar a un 30\% el reemplazo del agregado grueso natural por CCB.

Una resistencia mínima de 17,0 MPa para los concretos es estipulada en la normativa colombiana de construcción sismo-resistente (NSR10, 2010, Tabla C.4.3.1) [19] cuando se presentan las mejores condiciones ambientales (es decir, donde el ambiente es seco o protegido de la humedad, que no está expuesto a ciclos de congelamiento y deshielo, no se requiere baja permeabilidad y la concentración de sulfatos solubles en agua en contacto con el concreto es baja) y para aplicaciones en estructuras con capacidad de disipación de energía mínima. 
Considerando lo anterior y los resultados reportados en la Tabla 1, las futuras investigaciones deberían considerar en el diseño de la mezcla de concreto una cantidad de cemento mayor a $250 \mathrm{Kg}$ y una relación $\mathrm{A} / \mathrm{C}$ menor a 0,70 .

Resistencia a la compresión del concreto con bloque de arcilla triturado como agregado grueso: un caso práctico.

Se extrajo el segmento superior de una columna interior de un establecimiento penitenciario con una sección transversal de 250x250 mm (Figura 2), este establecimiento se encuentra en un nivel de agresividad III de acuerdo con la NTC-5551 (2007) [20], una temperatura promedio de $30^{\circ} \mathrm{C}$, humedad relativa de $80 \%$ aproximadamente y una altura de 3 msnm. Como se observa en la Figura 2-c, el agregado grueso es reemplazado por triturado de ladrillo de arcilla.

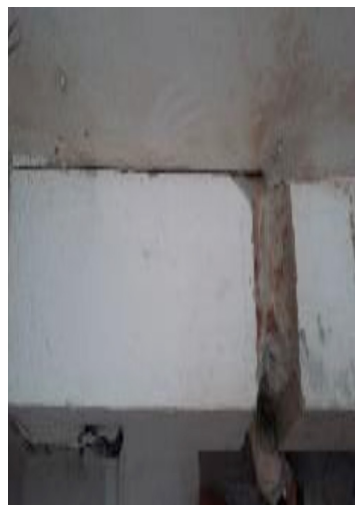

a) Localización de la columna

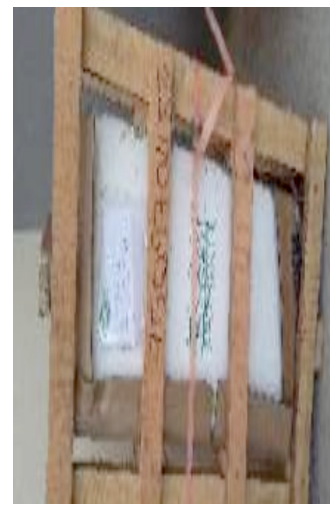

b) Sección de Columna de concreto extraída

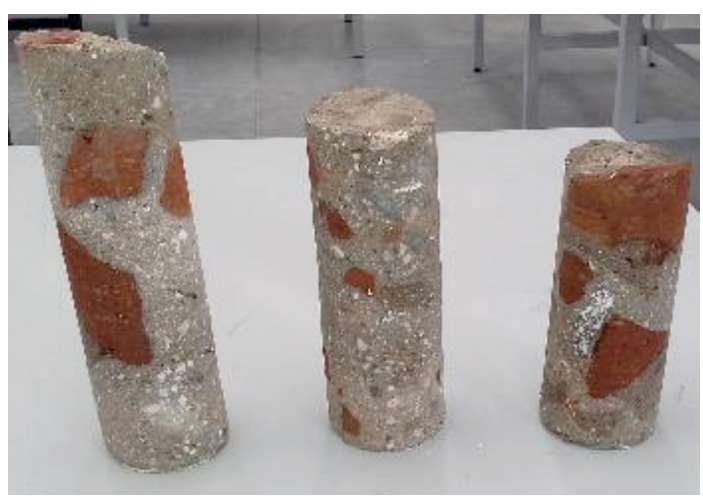

c) Núcleos extraídos

Figura 2. Muestra de concreto endurecido (Segura y Cardona, 2016).
Tres núcleos fueron extraídos (Figura 2-c) usando la metodología de la norma NTC-3658 [21] y sometidos al ensayo de compresión de acuerdo con la norma INVIAS [22]. La resistencia de estos cilindros fueron de 6,6, 7,6 y 10,8 MPa [23]. Es decir, una resistencia promedio de 8.3 MPa que se encuentra por debajo de los valores promedio reportados por otros investigadores (ver Tabla 1) y de lo especificado por la normativa colombiana (17.0 MPa, NSR10, 2010), esto puede deberse a que el tamaño del agregado de ladrillo triturado es mucho mayor que el tamaño máximo del agregado grueso triturado que se usa en una mezcla de concreto estructural (es decir, 3/4 de pulgada) y/o una posible relación $\mathrm{A} / \mathrm{C}$ muy alta (ejemplo, mayor a 0,7 ).

\section{AGRADECIMIENTOS}

Los autores expresan los agradecimientos a la Universidad Militar Nueva Granada por el apoyo al proyecto de investigación INV-DIS-2323 "Estudio del efecto de la corrosión del acero de refuerzo en elementos estructurales construidos en concreto bajo los criterios de la norma sismo resistente colombiana 10 título c"; de este proyecto se derivó este artículo.

\section{CONCLUSIONES}

Aunque existe una gran variedad de literatura publicada sobre el uso de productos dela demolición $[4,7,24]$, son escasos los resultados publicados sobre el efecto neto del uso de triturado de bloque de arcilla como agregado grueso en las diferentes propiedades del concreto tanto en su estado fresco como endurecido. En este sentido, una gran cantidad de estudios son sugeridos en el futuro, esto con la finalidad de establecer lineamientos más claros sobre el diseño de este tipo de concretos. Además, estos estudios deben incorporar cementos diferentes al cemento portland ordinario tipo o de uso general.

Considerando que el agregado $\mathrm{CCB}$ reduce tanto las características mecánicas y como de durabilidad de los concretos comparado con los agregados naturales [1]. Establecer límites de reemplazo en los porcentajes de reemplazo de este agregado es apenas necesario para algunas aplicaciones estructurales. Basado en la revisión de literatura, se recomienda ampliar la cantidad de investigaciones en mezclas de concreto con una cantidad de cemento mayor a $250 \mathrm{Kg}$ y una relación A/C menor a 0,70 . 
En cuanto a la resistencia a la compresión, se reafirma que una reducción promedio del $30 \%$ es estimada al reemplaza el $100 \%$ de los agregados gruesos por el bloque de arcilla triturado dentro de las relaciones A/C reportadas en la literatura (entre 0,46 y 0,74 ). Precauciones especiales deben considerarse al tratar de utilizar este tipo de agregados en ambientes con presencia de concentración de cloruros en el ambiente (e.g. zonas costeras) debido a la alta porosidad y permeabilidad que exhibe este tipo de mezclas de concreto.

\section{REFERENCIAS}

[1] A.A. Aliabdo, A.E.M. Abd-Elmoaty and H.H. Hassan. "Utilization of crushed clay brick in concrete industry". Alexandria Engineering Journal. Vol. 53 No 1, pp. 151-168. 2014.

[2] M. Pepe, R.D. Toledo Filho, E.A. Koenders and E. Martinelli. "Alternative processing procedures for recycled aggregates in structural concrete". Construction and Building Materials. Vol. 69, pp. 124-132. 2014.

[3] A. Sagastume, J. Cabello, C. Gaviria, J. Caneghem and C. Vandecasteele. "Improved selection of the functional unit in the environmental impact assessment of cement". Journal of Cleaner Production, In-press. 2017.

[4] N. Kisku, H. Joshi, M. Ansari, S. K. Panda, S. Nayak and S.C. Dutta. "A critical review and assessment for usage of recycled aggregate as sustainable construction material". Construction and Building Materials. Vol. 131, pp. 721-740. 2017.

[5] M. Ospina, L. Moreno and K. Rodriguez. "Análisis Técnico-Económico del Uso de Concreto Reciclado y el Concreto Convencional en Colombia". En Desarrollo e Innovación en Ingeniería, 722 p. Capítulo 46, pp. 656-673. Agosto 2017.

[6] W. Martínez-Molina, A.A. Torres-Acosta, E.M. Alonso-Guzmán, H.L. Chávez-García, H. Hernández-Barrios, C. Lara-Gómez, W. Martínez-Alonso, J.T. Pérez-Quiroz, J.A. Bedolla-Arroyo and F.M. González-Valdéz. "Concreto reciclado: una revisión". Revista ALCONPAT. Vol. 5 No 3, pp. 235-248. 2015.

[7] A.E.B. Cabral, V. Schalch, D.C.C. Dal Molin and J.L.D. Ribeiro. "Mechanical properties modeling of recycled aggregate concrete". Construction and Building Materials. Vol. 24 $\mathrm{N}^{\circ}$ 4, pp. 421-430. 2010.
[8] M. Adamson, A. Razmjoo and A. Poursaee. "Durability of concrete incorporating crushed brick as coarse aggregate". Construction and Building Materials. Vol. 94, pp. 426-432. 2015.

[9] F. Pacheco-Torgal and S. Jalali. "Reusing ceramic wastes in concrete". Construction and Building Materials. Vol. $24 \mathrm{~N}^{\circ}$ 5, pp. 832838. 2010

[10] F. Debieb and S. Kenai. "The use of coarse and fine crushed bricks as aggregate in concrete". Construction and building materials. Vol. 22 $\mathrm{N}^{\circ}$ 5, pp. 886-893. 2008.

[11] J. Yang, Q. Du and Y. Bao. "Concrete with recycled concrete aggregate and crushed clay bricks". Construction and Building Materials. Vol. $25 \mathrm{~N}^{\circ}$ 4, pp. 1935-1945. 2011.

[12] S.I. Ahmad and S. Roy. "Effect of Crushed Clay Brick as Coarse Aggregate on Creep Behavior of Concrete". In Advanced Materials Research. Vol. 261, pp. 178-181. Trans Tech Publications. 2011.

[13] Á.V. Pérez-Rojas. "Uso de triturado de ladrillo reciclado como agregado grueso en la elaboración de concreto". Ingenium Revista de la facultad de ingeniería. Vol. $13 \mathrm{~N}^{\circ} 26$, pp. 116-125. 2012.

[14] Y. Silva, R. Robayo, P. Mattey and S. Delvasto. "Obtención de concretos autocompactantes empleando residuos de demolición”. Revista Latinoamericana de Metalurgia y Materiales. Vol. $35 \mathrm{~N}^{\circ}$ 1, pp. 86-94. 2015.

[15] I. Martínez-Lage, F. Martínez-Abella, C. Vázquez-Herrero and J.L. Pérez-Ordóñez. "Properties of plain concrete made with mixed recycled coarse aggregate". Construction and Building Materials. Vol. 37, pp. 171-176. 2012.

[16] F.S. Kallak. "Use of crushed bricks as coarse aggregate in concrete". Tikrit Journal of Engineering Science (TJES). Vol. $16 \mathrm{~N}^{\mathrm{o}} 3$, pp. 64-69. 2009.

[17] V.A. Ulloa, E. García-Taengua, M.J. Pelufo, A. Domingo and P. Serna. "New views on effect of recycled aggregates on concrete compressive strength". ACI Materials Journal. Vol. $110 \mathrm{~N}^{\circ}$ 6, pp. 1-10. 2013.

[18] T.C. Hansen. "Recycling of demolished concrete and masonry". RILEM Report No 6, CRC Press, 336 p. 1992.

[19] Asociación Colombiana de Ingeniería Sísmica. AIS. "Reglamento colombiano de Construcción Sismo Resistente (NSR10)", Colombia. Bogotá, DC. 2010. 
[21] Norma técnica colombiana (NTC-3658) "Ingeniería civil y arquitectura. Método para la obtención y ensayo de núcleos extraídos y vigas de concreto aserradas", Bogotá, Icontec, 1994.

[20] Norma técnica colombiana (NTC-5551). "Concretos: Durabilidad de estructuras de concreto". Bogotá, Icontec, 2007.

[22] INV-E-410-13. "Resistencia a la compresión de cilindros de concreto". Bogotá, Invias, 2013.

[23] C.A. Segura y P.A. Cardona. "Determinación de los procesos de corrosión del acero en las estructuras de concreto en el establecimiento penitenciario de mediana seguridad y carcelario de carcelario Ciénaga Magdalena”. Trabajo de Grado, Universidad Militar Nueva Granada, 115 páginas. 2016.

[24] M. Behera, S.K. Bhattacharyya, A.K. Minocha, R. Deoliya and S. Maiti. "Recycled aggregate from $C \& D$ waste $\&$ its use in concrete-A breakthrough towards sustainability in construction sector: A review". Construction and building materials. Vol. 68, pp. 501-516. 2014. 